\title{
[CII] emission properties of the massive star-forming region RCW 36 in a filamentary molecular cloud
}

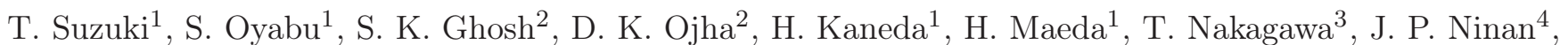 \\ S. Vig $\stackrel{5}{5}$, M. Hanaokd ${ }^{1}$, F. Saitd ${ }^{1}$, S. Fujiward ${ }^{1}$, and T. Kanayama ${ }^{1}$
}

1 Graduate School of Science, Nagoya University, Furo-cho, Chikusa-ku, Nagoya, Aichi, 464-8602, Japan
2 Tata Institute of Fundamental Research, Homi Bhabha Road, Colaba, Mumbai 400005, India
3 Institute of Space and Astronautical Science, Japan Aerospace Exploration Agency 3-1-1 Yoshinodai, Chuo-ku,
Sagamihara, Kanagawa, 252-5210, Japan
4 The Pennsylvania State University, University Park, State College, PA, USA
5 Indian Institute of Space Science and Technology, Valiamala, Thiruvananthapuram 695 547, India

Received / Accepted

\begin{abstract}
Aims. To investigate properties of [C II] $158 \mu \mathrm{m}$ emission of RCW 36 in a dense filamentary cloud.

Methods. [C II] observations of RCW 36 covering an area of $\sim 30^{\prime} \times 30^{\prime}$ were carried out with a Fabry-Pérot spectrometer aboard a $100-\mathrm{cm}$ balloon-borne far-infrared (IR) telescope with an angular resolution of 90". By using AKARI and Herschel images, the spatial distribution of the [C II] intensity was compared with those of emission from the large grains and polycyclic aromatic hydrocarbon $(\mathrm{PAH})$.

Results. The [C II] emission is spatially in good agreement with shell-like structures of a bipolar lobe observed in IR images, which extend along the direction perpendicular to the direction of a cold dense filament. We found that the [C II $]-160 \mu \mathrm{m}$ relation for RCW 36 shows higher brightness ratio of $[\mathrm{C} \mathrm{II}] / 160 \mu \mathrm{m}$ than that for $\mathrm{RCW} 38$, while the [C II] $-9 \mu \mathrm{m}$ relation for RCW 36 is in good agreement with that for RCW 38.

Conclusions. By spectral decomposition analysis on a pixel-by-pixel basis using IR images, the [C II] emission spatially well correlates with PAH and cold dust emissions. This means that the observed [C II] emission dominantly comes from PDRs. Moreover, the $L_{\left[\mathrm{C}_{\mathrm{II}}\right]} / L_{\mathrm{FIR}}$ ratio shows large variation $\left(10^{-2}-10^{-3}\right)$ compared with the $L_{\left[\mathrm{C}_{\mathrm{II}}\right]} / L_{\mathrm{PAH}}$ ratio. In view of the observed tight correlation between $L_{[\mathrm{C} \text { II }} / L_{\mathrm{FIR}}$ and the optical depth at $\lambda=160 \mu \mathrm{m}$, the large variation in $L_{[\mathrm{C} \text { II }} / L_{\mathrm{FIR}}$ can be simply explained by the geometrical effect, viz., $L_{\mathrm{FIR}}$ has contributions from the entire dust-cloud column along the line of sight, while $L_{[\mathrm{C} \text { II }}$ has contributions from far-UV illuminated cloud surfaces. Based on the picture of the geometry effect, the enhanced brightness ratio of $[\mathrm{CII}] / 160 \mu \mathrm{m}$ is attributed to the difference in gas structures where massive stars are formed: filamentary (RCW 36) and clumpy (RCW 38) molecular clouds and thus suggests that RCW 36 is dominated by far-UV illuminated cloud surfaces compared with RCW 38.
\end{abstract}

Key words. ISM:dust - infrared:ISM - ISM:lines and bands - ISM:H II regions - ISM:individual objects: RCW 36

\section{Introduction}

Understanding of galaxy evolution remains a key subject in modern astrophysics. Massive stars $\left(\gtrsim 8 M_{\odot}\right)$ have a great influence on the evolution of the interstellar medium (ISM) energetically, chemically, and dynamically, and thus plays a vital role in galaxy evolution. More recently, Herschel observations reveal the presence of ubiquitous filamentary structures connecting Galactic star-forming regions with each other, suggesting that filaments have the crucial role on star formation (e.g. Molinari et al. 2010; André et al. 2010). Special attention on filament structures has been promoting investigations of the radiative feedback of massive stars on their filamentary ISM, which regulates subsequent star formation as well as the role of filaments in the formation of dense star-forming clumps (Zinnecker \& Yorke 2007; Mvers 2009; André et al. 2010; Baug et al. 2015; Dewangan et al. 2017a, b).

A photo-dissociation region (PDR) is formed around an $\mathrm{H}$ II region by massive stars, and is the region where farultraviolet (UV) $(6<h \nu<13.6 \mathrm{eV})$ photons play a signifi- cant role in the heating and chemistry of the ISM; all of the atomic and at least $90 \%$ of the molecular gas in the Milky Way are in PDRs (Hollenbach \& Tielens 1999). This means that PDRs are key to understanding the physical properties of the ISM strongly influenced by the radiative feedback of massive stars, and the triggering of next-generation stars. In PDRs, the gas heating is mainly caused by photoelectrons (photoelectric heating) from dust grains and polycyclic aromatic hydrocarbons (PAHs), which are ejected by absorbing far-UV photons from stars, while the gas cooling is performed by far-infrared (IR) fine-structure lines, among which [C II] $158 \mu \mathrm{m}$ is the most important gas coolant in low-density PDRs (e.g. Hollenbach \& Tielens 1999).

Studies of gas cooling and photo-electric heating of gas in PDRs require [C II] and IR photometric data sets and sufficient spatial resolution to trace the variety of dust emission components such as PAHs, warm $(\sim 60-100 \mathrm{~K})$, and cold ( $\sim 20-30 \mathrm{~K})$ dust emissions and to diagnose region-byregion physical conditions in the ISM. The reason is that gas heating and cooling processes depend on dust grain types 


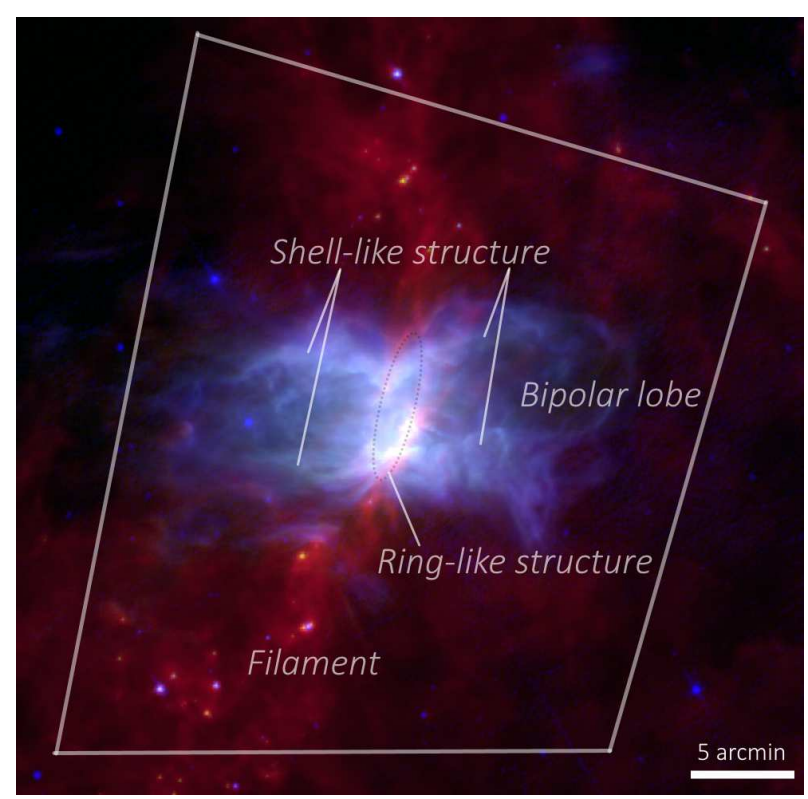

Fig. 1. [C II] observation area (solid box) of RCW 36 superimposed on a composite image shown with the equatorial J2000 coordinate system: AKARI $9 \mu \mathrm{m}$ (blue), Herschel $70 \mu \mathrm{m}$ (green), and Herschel $250 \mu \mathrm{m}$ (red). RCW 36 is formed in a large-scale filament extending north-south direction. A bipolar lobe, shelllike structures and a ring-like structure (black dashed ellipse) are identified by Minier et al. (2013).

and ISM conditions. Although Herschel and SOFIA enable us to have an opportunity to access such data sets for the first time, spatial coverage large enough to include an entire Galactic star-forming region remains poor for global understanding of PDRs, in particular for a [C II] map.

RCW 36 is a Galactic massive star-forming region formed in a filament structure of the Vela $\mathrm{C}$ molecular cloud complex (Hill et al. 2011); the H II region is formed by $\sim 350$ stars including two O-type and one B-type stars (Ellerbroek et al. 2013). Recent CO observations suggest that massive stars were formed by a cloud-cloud collision along the filament direction (Sano et al. 2018). The composite IR image in Fig. 1 shows two major features in addition to the filament structure: 1) a bipolar lobe with shell-like structures extending the east-west direction which is perpendicular to the filament structure, 2) a ring-like structure (dashed ellipse) with a major axis of $2 \mathrm{pc}$ at its center. The two features are attributed to blowout of the filament structure towards surrounding lowdensity regions because of intense ionizing radiation and stellar winds from massive stars (Minier et al. 2013). Thus, RCW 36 showing spectacular features is a best laboratory to understand the radiative feedback from massive stars. In this paper, the distance to RCW 36 is taken to be $1.09 \mathrm{kpc}$ (Gaia Collaboration et al. 2018).

\section{Observations and Data analysis}

\section{1. [CII] $158 \mu \mathrm{m}$ data from $\mathrm{T} 100$}

We carried out [CII] observations of RCW 36 on 15 Nov. 2004, 30 Nov. 2017, and 28 Oct. 2018 by a FabryPérot spectrometer aboard a 100-cm balloon-borne farIR telescope with an angular resolution of $90^{\prime \prime}$ (hereafter

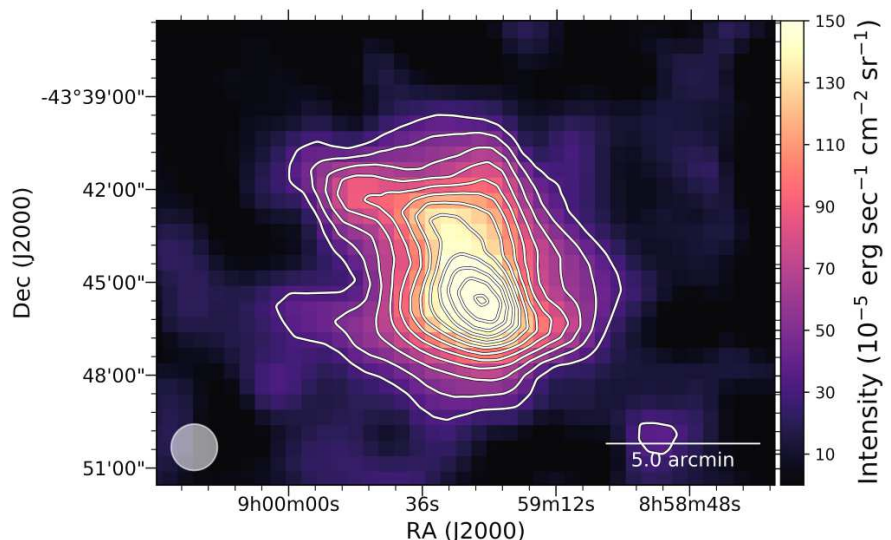

Fig. 2. [C II] intensity map of RCW 36 showing an area of $20^{\prime} \times$ $15^{\prime}$ taken by FPS100. The contours are linearly spaced 12 levels from $3.3 \times 10^{-4}$ to $1.9 \times 10^{-3} \mathrm{erg} \mathrm{sec}^{-1} \mathrm{~cm}^{-2} \mathrm{sr}^{-1}$. The PSF size in FWHM is shown in the lower left corner.

FPS100: Ghosh et al. 1988; Nakagawa et al. 1998). At the Hyderabad Balloon Facility of the Tata Institute of Fundamental Research (TIFR) in India, FPS100 was launched into the stratosphere of an altitude of $\sim 30 \mathrm{~km}$. RCW 36 was then observed with a spatially unchopped, fast spectral scan mode by two sets of the spatial raster scans covering a large-scale area of $\sim 30^{\prime} \times 30^{\prime}$ in total (Fig. 11); detailed descriptions on FPS100 and its observation modes can be found in Mookerjea et al. (2003). Telescope pointing to the science target was achieved by offsetting it with reference to a nearby bright guide star and the absolute pointing error is estimated to be typically $\sim 60^{\prime \prime}$. [C II] flux calibrations were made with Orion B by comparing its [C II] map taken by Kuiper Airborne Observatory (Jaffe et al. 1994); the flux calibration error is $\sim 10 \%$.

The data reduction follows the same manner as Kaneda et al. (2013); a [C II] intensity map was obtained by subtracting atmospheric background and astronomical continuum emission components from each spectrum scan. For each set of the spatial scans taken in 2004, 2017, and 2018, an atmospheric background spectrum was estimated by averaging the spectral scan data at the eastern edge of the spatial scan legs every three legs; an area of $\sim 30^{\prime} \times 7^{\prime}$ was used as the background region where no significant $[\mathrm{C} I \mathrm{I}]$ line emission from RCW 36 was detected. Then, we fitted the spectral scan data on the corresponding spatial scans by a combination of the atmospheric background spectrum, a Rayleigh-Jeans regime modified black-body function with an emissivity power-law index $(\beta)$ of 2.0 for an astronomical $158 \mu \mathrm{m}$ continuum emission, and a Lorentzian function for the [C II] emission. Amplitudes of the atmospheric background, the astronomical $158 \mu \mathrm{m}$ continuum, and [C II] emission components were set to be free, while the center and the width of the Lorentzian function were fixed to the values estimated from the data of several spectral scans with high signal-to-noise ratio. To determine the positional offset in a [C II] map, we shifted the $158 \mu \mathrm{m}$ continuum emission map along the R.A. and Dec. axes so that the peak in the continuum emission map is matched to that in a Herschel $160 \mu \mathrm{m}$ map. Finally, the combined [C II] map obtained from the three maps was regridded with pixel sizes of $90^{\prime \prime}$ for pixel-by-pixel correlation analyses. This ensures measurements for every pixel to be independent samples (without coupling with the neighbouring pixels). 


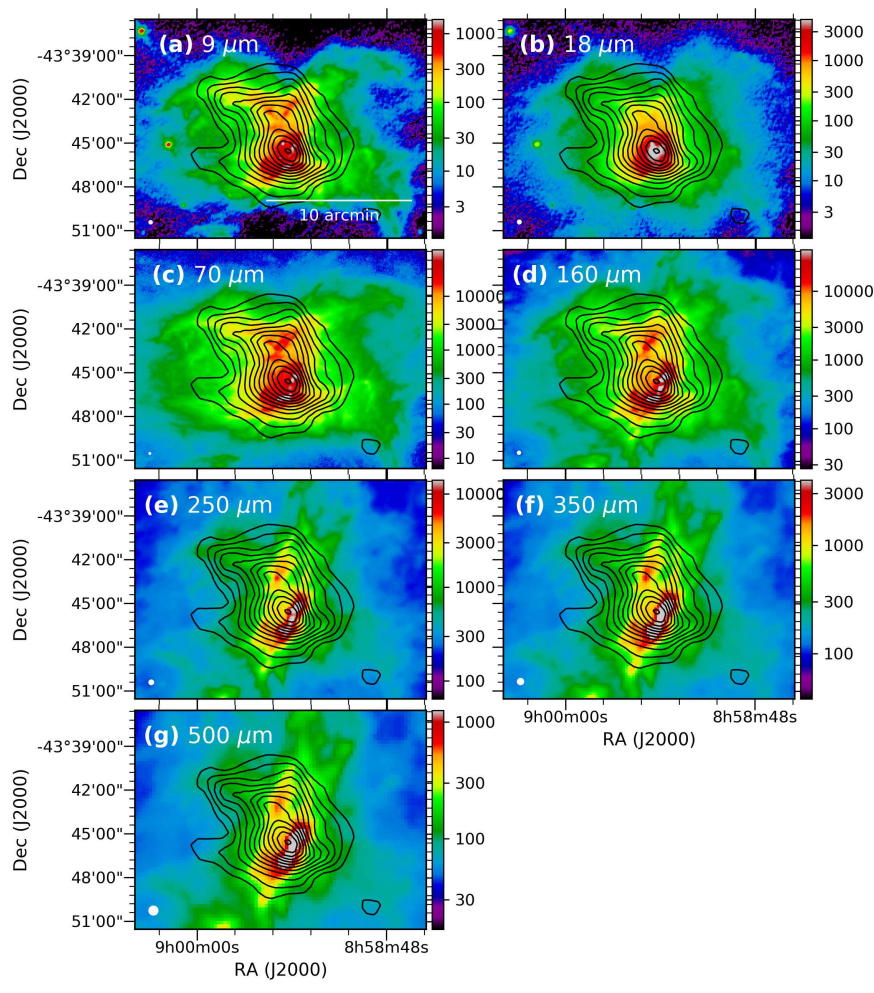

Fig. 3. Seven-band images of RCW 36 from the AKARI and Herschel data in (a) AKARI $9 \mu \mathrm{m}$, (b) AKARI $18 \mu \mathrm{m}$, (c) Herschel $70 \mu \mathrm{m}$, (d) Herschel $160 \mu \mathrm{m}$, (e) Herschel $250 \mu \mathrm{m}$, (f) Herschel $350 \mu \mathrm{m}$, and (g) Herschel $500 \mu \mathrm{m}$ bands. In each image, the color bar is given in units of $\mathrm{MJy} \mathrm{sr}^{-1}$. The PSF size in FWHM is in the lower left corner. The contours superimposed on the images are the same as those in Fig 2 .

\subsection{Broad-band IR data from AKARI and Herschel}

RCW 36 was observed with the AKARI all-sky survey in mid-IR wavelengths (Onaka et al. 2007; Ishihara et al. 2010) and Herschel as part of HOBYS (The Herschel imaging survey of OB young stellar objects) guaranteed time key program (Hill et al. 2011). For comparison with the [C II] map, we used two-band images from AKARI (9 and $18 \mu \mathrm{m})$ and five-band images from Herschel (70, 160, 250, 350, and $500 \mu \mathrm{m})$.

The spatial resolutions of AKARI $9 \mu \mathrm{m}$ and $18 \mu \mathrm{m}$ are 12 and $14^{\prime \prime}$, respectively. The flux calibration uncertainty of the two bands is $\sim 10 \%$ (Ishihara et al. in prep.). The Herschel data were taken from the Herschel Science Archive. The Herschel/PACS and SPIRE data are level2.5 products (SPG version 14.2.0) providing JScanam maps and level-2 products (SPG version 14.1.0) providing Naive maps, respectively. The beam size has the FWHMs of 5 , 11, 18, 24, and $35^{\prime \prime}$ at Herschel $70 \mu \mathrm{m}, 160 \mu \mathrm{m}, 250 \mu \mathrm{m}$, $350 \mu \mathrm{m}$, and $500 \mu \mathrm{m}$, respectively. The systematic flux calibration uncertainty of the Herschel bands is applied to be $\sim 5 \%$ (PACS Observer's Manual version 2.5.1 and SPIRE Observer's Manual version 2.5).

A background level for each of the seven-band IR images was estimated by averaging pixel values in a region where no significant [CII] emission was detected to subtract a diffuse component that is not associated to RCW 36 from each image. The background level is $0.6 \%$ (AKARI $9 \mu \mathrm{m}), 0.2 \%$ (AKARI $18 \mu \mathrm{m}$ ), 0.1\% (Herschel $70 \mu \mathrm{m})$, $0.4 \%$ (Herschel $160 \mu \mathrm{m}$ ), 3\% (Herschel $250 \mu \mathrm{m}$ ), 4\% (Her-
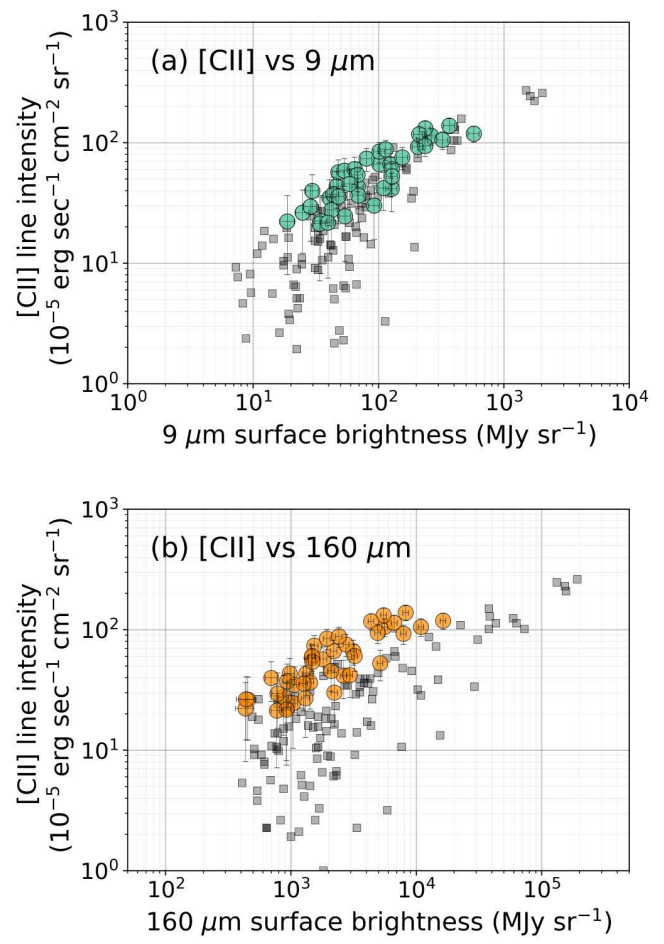

Fig. 4. Correlation plots of brightness between (a) [C II] and $9 \mu \mathrm{m}$, and (b) [C II] and $160 \mu \mathrm{m}$ for RCW 36 (circle) together with those for RCW 38 (square) from Fig.3 in Kaneda et al. (2013).

schel $350 \mu \mathrm{m}$ ), and $5 \%$ (Herschel $500 \mu \mathrm{m}$ ) of the peak surface brightness. For multi-band analysis, the spatial resolutions of background-subtracted IR images were reduced to match a Gaussian PSF with the FWHM of $90^{\prime \prime}$ for the FPS100 data by convolving PACS and SPIRE-band images with kernels provided by Aniano et al. (2011). Because such kernels for the AKARI bands are currently not available in the library 1 , the AKARI images were convolved by a Gaussian kernel to approximate the FPS100 PSF. Then, the convolved images were regridded with a pixel size of $90^{\prime \prime}$ matched with the PSF of the FPS100 data for pixel-bypixel correlation analyses.

\section{Results}

\section{1. [CII] $158 \mu \mathrm{m}$ and broadband IR maps}

Figure 2 shows the $[\mathrm{CII}]$ intensity map of RCW 36 by FPS100. The 1-sigma fluctuation per pixel is $1.1 \times 10^{-4}$ erg sec ${ }^{-1} \mathrm{~cm}^{-2} \mathrm{sr}^{-1}$. The [C II] emission overall extends toward the east-west direction from the peak, which is almost perpendicular to the direction of the filament structure as shown in Fig. 1 .

The seven-band IR images by AKARI and Herschel are shown in Fig. 3. The AKARI $9 \mu \mathrm{m}$ image mainly traces emission from PAHs and shows a bipolar lobe with shell-like structures in the east-west direction (Fig. 1). The overall spatial distribution of the AKARI $9 \mu \mathrm{m}$ map is in good agreement with that of the Herschel 70-500 $\mu \mathrm{m}$ maps which trace emission from large grains, although the Herschel 250-500 $\mu \mathrm{m}$ maps show more prominent filament structure in addition to the bipolar lobe. In the

${ }^{1}$ http://www.astro.princeton.edu/ ganiano/Kernels.html 


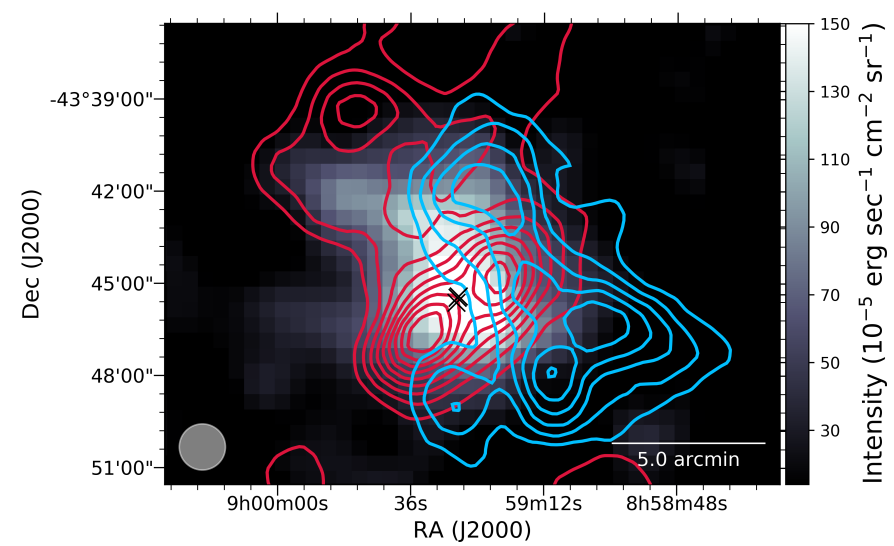

Fig. 5. Same as Fig. 2, but ${ }^{12} \mathrm{CO}(J=2-1)$ contours (Fig. 4a in Sano et al. 2018) and positions of OB stars (cross) (Ellerbroek et al. 2013). The cyan and red colored contours represent two-velocity molecular cloud components with $V_{\mathrm{LSR}}=4.1-6.1 \mathrm{~km} \mathrm{~s}^{-1}$ (blue cloud) and $7.6-12.0 \mathrm{~km} \mathrm{~s}^{-1}$ (red cloud), respectively. The spatial resolution of the ${ }^{12} \mathrm{CO}(J=2-1)$ map taken by NANTEN2 is similar to the $[\mathrm{C} \mathrm{II}]$ $\operatorname{map}\left(90^{\prime \prime}\right)$

AKARI $18 \mu \mathrm{m}$ image which traces emissions from large and very small grains, the spatial distribution also shows the bipolar lobe but does not show shell-like structures clearly.

To compare spatial distributions between the [C II] map and a broadband IR emission map, contours of the [C II] intensity are superimposed on each IR map (Fig. 3). The [C II] map shows two prominent arms extending from its peak to the north-east and south-west directions, which are spatially in good agreement with shell-like structures of the bipolar lobe observed in the broadband IR images. The peak position in the $[\mathrm{CII}]$ map is located close to the IR bright rims along the ring-like structure Minier et al. 2013). For quantitative comparison of spatial distributions, Fig. 4 shows pixel-by-pixel correlation plots of (a) [C II] vs. $9 \mu \mathrm{m}$ and (b) [C II] vs. $160 \mu \mathrm{m}$. Here, we removed pixels with the optical depth at $\lambda=9 \mu \mathrm{m}$ higher than unity from correlation plots. Since the AKARI $9 \mu \mathrm{m}$ band is overlapping with the interstellar silicate feature around $9.7 \mu \mathrm{m}$, the interstellar extinction in the AKARI $9 \mu \mathrm{m}$ band is the most severe among the seven IR bands. To evaluate the extinction, the optical depth at $\lambda=9 \mu \mathrm{m}, \tau_{9}$ is calculated from $\tau_{9}=4.8 \times 10^{-2} A_{\mathrm{V}}$ by applying $A_{9} / A_{\mathrm{Ks}}=5.8 \times$ $10^{-1}$ (Xue et al. 2016) and $A_{\mathrm{Ks}} / A_{\mathrm{V}}=8.9 \times 10^{-2}$ (Glass 1999); $\tau_{9}>1$ corresponds to $A_{\mathrm{V}}>21$ mag. Hill et al. (2011) investigated an $N_{\mathrm{H}_{2}}$ column density map of the Vela C molecular complex including RCW 36 based on pixel-by-pixel SED fitting in the wavelength range of 70 $500 \mu \mathrm{m}$. We then converted the $N_{\mathrm{H}_{2}}$ column density to visual extinction units assuming $N_{\mathrm{H}_{2}}=0.94 \times 10^{21} A_{\mathrm{V}}$ $\mathrm{cm}^{-2}$ Bohlin et al. 1978). From the map around RCW 36, the regions with $A_{\mathrm{V}}>21 \mathrm{mag}$ are located around the peak seen in the Herschel $500 \mu \mathrm{m}$ map. Therefore, the relevant pixels showing $\tau_{9}>1$ were removed. Clearly, the [C II]$9 \mu \mathrm{m}$ relation for RCW 36 is in good agreement with that for RCW 38 as denoted by black-filled squares, while the [C II]-160 $\mu \mathrm{m}$ relation for RCW 36 shows higher brightness ratio of $[\mathrm{C} I \mathrm{II}] / 160 \mu \mathrm{m}$ than that for $\mathrm{RCW} 38$.

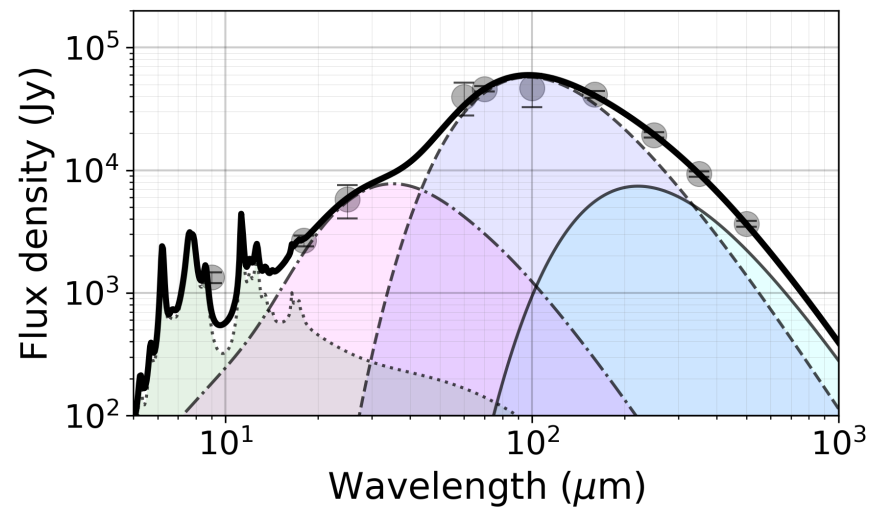

Fig. 6. Global SED of the whole region of RCW 36 obtained from the aperture radius of $25^{\prime}$ at the center position of $\mathrm{RA}=$ 8:59:30.963, Dec $=-43: 46: 56.270$ (J2000.0). To obtain the eightfree parameters, the SED fitting was performed for the original seven-band data plus IRAS $25 \mu \mathrm{m}, 60 \mu \mathrm{m}$, and $100 \mu \mathrm{m}$ data. The thick solid line shows the best-fitting model, which is described in Eq. (1). The very cold dust, cold dust, warm dust, and PAH components are denoted by thin solid, dashed, dash-dotted, and dotted lines, respectively.

\section{2. [CII] $158 \mu \mathrm{m}$ and molecular gas maps}

Sano et al. (2018) presented the latest CO observations of RCW 36 and found two molecular clouds at the velocities $V_{\mathrm{LSR}} \sim 5.5 \mathrm{~km} \mathrm{~s}^{-1}$ (blue cloud) and $9 \mathrm{~km} \mathrm{~s}^{-1}$ (red cloud), which are likely to be physically associated with RCW 36. They also showed that there is no bridge-like feature connecting the two molecular clouds in velocity space. Figure 5 shows ${ }^{12} \mathrm{CO}(J=2-1)$ contours (Fig. 4a in Sano et al. 2018) overlaid on the [C II] image. The [C II] emission is not spatially in good agreement with the ${ }^{12} \mathrm{CO}$ emission for both red and blue cloud components; the bright ${ }^{12} \mathrm{CO}$ emission from the dense red cloud component has the double peak and its structure is elongated along the filament structure. The double peak is located around the [C II] peak and the massive stars as denoted by the cross marks. Sano et al. (2018) found that the dense red cloud coincides with the star cluster, while the diffuse blue cloud does not. The red cloud seems to be physically close to the star cluster and its proximate surface ionized by the UV radiation from these stars, while the blue cloud is less affected by UV from this cluster due to its far location. Moreover, the diffuse CO emission extending from the filament structure to eastern (red cloud) and western (blue cloud) directions tends to be distributed in the outer rim of shell-like structures seen in [C II] and broadband IR maps.

From the above situation in spatial distributions, we propose a possible geometry of the $[\mathrm{C}$ II] emission region with respect to the two collided molecular clouds as follows: for the positional relation among the observer, the red cloud, and the blue cloud, there are two possible cases. The first case is that the red cloud is closer to the observer when the red and blue clouds are colliding with each other, while the second case is that the blue cloud is closer to the observer when the red cloud went through the blue one after collision. As shown in Fukui et al. (2016), colliding two clouds are generally connected to each other in velocity space and show a bridge-like feature in a position-velocity diagram. Because the bridge-like feature was not observed in RCW36 (Sano et al. 2018), the observational result supports the second case. Therefore, the dense red cloud with 

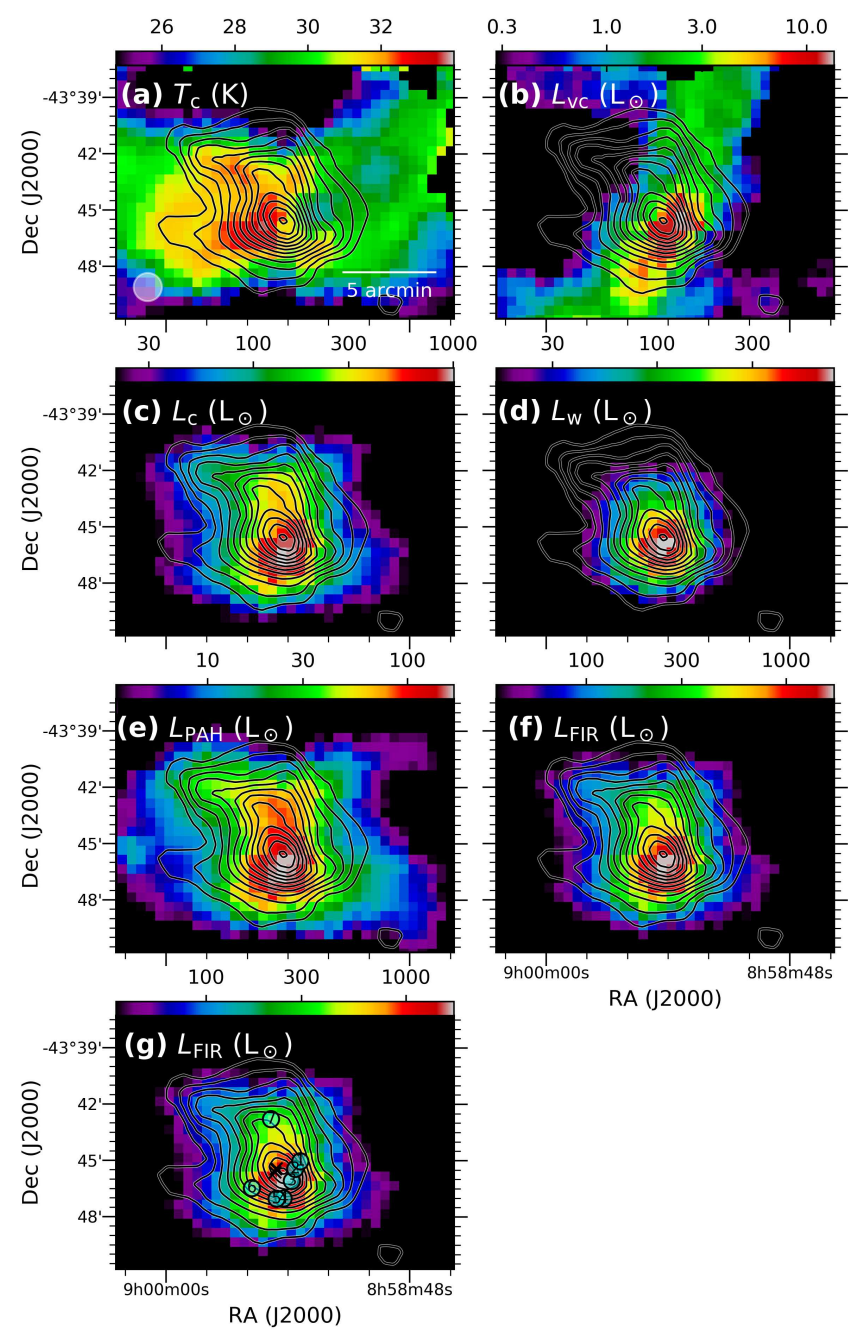

RA (J2000)

Fig. 7. Spatial distributions of (a) cold dust temperature $\left(T_{\mathrm{c}}\right)$, (b) very cold dust luminosity $\left(L_{\mathrm{vc}}\right)$, (c) cold dust luminosity $\left(L_{\mathrm{c}}\right),(\mathrm{d})$ warm dust luminosity $\left(L_{\mathrm{w}}\right),($ e) PAH luminosity $\left(L_{\mathrm{PAH}}\right)$, and (f) far-IR luminosity $\left(L_{\mathrm{FIR}}=L_{\mathrm{vc}}+L_{\mathrm{c}}+L_{\mathrm{w}}\right)$. The panel $(\mathrm{g})$ is same as the panel (f), but the positions of seven clumps and OB stars are denoted by circles and crosses, respectively (Ellerbroek et al. 2013; Minier et al. 2013). Dust temperature and luminosity are in units of kelvin and solar luminosity, respectively. For each luminosity map, the color scale ranges from $2 \%$ to $98 \%$ of the peak luminosity. The contours superimposed on the images are the same as those in Fig. 2

the molecular filament went through the blue cloud, and is then associated with the massive stars formed by the cloud-cloud collision, while the diffuse red and blue clouds are mostly located the outer rim of shell-like structures. The massive stars formed by the collision between the red and blue clouds illuminate surface of those two clouds. Given that the most of the $[\mathrm{C} \mathrm{II}]$ emission originates from those far-UV illuminated surface of clouds, the [C II]-emitting region is expected to be facing toward observers without significant attenuation by foreground dust grains associated with CO clouds.

\section{Discussion}

\subsection{Spectral decomposition into very cold dust, cold dust, warm dust and $\mathrm{PAH}$ components}

Spectral decomposition analysis on a pixel-by-pixel basis enables us to investigate spatial distributions of PAH and dust properties and the relation between those properties and [CII] emission. An individual SED constructed from the seven-band fluxes at each pixel (pixel size of $90^{\prime \prime}$ ) is reproduced by a triple-component modified blackbody plus a PAH model expressed as

$$
\begin{aligned}
F_{\nu, \mathrm{IR}}= & A_{\mathrm{PAH}} F_{\nu, \mathrm{PAH}}+A_{\mathrm{vc}} \nu^{\beta_{\mathrm{vc}}} B_{\nu}\left(T_{\mathrm{vc}}\right)+A_{\mathrm{c}} \nu^{\beta_{\mathrm{c}}} B_{\nu}\left(T_{\mathrm{c}}\right) \\
& +A_{\mathrm{w}} \nu^{\beta_{\mathrm{w}}} B_{\nu}\left(T_{\mathrm{w}}\right)\left\{1+\left(\frac{\nu_{c}}{\nu}\right)^{3} \nu^{-\alpha} e^{-\left(\frac{\nu_{c}}{\nu}\right)^{2}}\right\},
\end{aligned}
$$

where $T, \beta, A$, and $B_{\nu}(T)$ are the dust temperature, the dust emissivity power-law index, amplitude, and the Planck function, respectively. Suffixes vc, c, w, and PAH denote very cold dust, cold dust, warm dust and PAH components, respectively. The $\beta$ value for each dust component is assumed to be 2.0 as a typical value of the Galactic ISM (Anderson et al. 2012). The second term in the warm dust component is the analytic approximation of thermal emission from dust grains which are exposed to a range of starlight intensities: dust emissions with different dust temperatures assuming a power-law temperature distribution to take the hot dust component into account in midIR wavelengths (Casey 2012). The power-law turnover frequency $\nu_{c}$ defined by Casey (2012) is a function of the mid-IR power-law slope $\alpha$ and $T_{\mathrm{w}}$. The flux density of the PAH component, $F_{\mathrm{PAH}}(\nu)$, is calculated as described in Suzuki et al. (2010) and is based on the PAH parameters taken from Li \& Draine (2001) and Draine \& Li (2007) by assuming the PAH size distribution ranging from 3.55 to $300 \AA$, the fractional ionization and the temperature probability distribution for the typical diffuse ISM with the interstellar radiation field in the solar neighborhood. PAHs with sizes larger than $15 \AA$ contribute to $\sim 20 \mu \mathrm{m}$ continuum emission (Draine \& Li 2007). Since very small grains (VSGs) which are stochastically heated by absorbed farUV photons contribute to $\sim 20 \mu \mathrm{m}$ continuum emission, the PAH component in Eq. (11) takes the VSG emission into account.

The eight-free parameters for the SED model cannot be determined from a seven-band data set. We then fixed $T_{\mathrm{vc}}$ and $\alpha$ values obtained from a global SED of RCW 36 by adding IRAS $25 \mu \mathrm{m}, 60 \mu \mathrm{m}$, and $100 \mu \mathrm{m}$ data. Since the parameters $T_{\mathrm{w}}$ and $A_{\mathrm{w}}$ are both determined by the $18 \mu \mathrm{m}$ flux density, they are fully degenerate in the model. To avoid this degeneracy in the calculation of the spatial distribution of luminosity for the warm dust component, we adopted a fixed $T_{\mathrm{w}}$ value obtained from the global SED fitting. Fig. 6] shows the global SED together with the bestfit model. The best-fit parameters of $T_{\mathrm{vc}}, T_{\mathrm{c}}, T_{\mathrm{w}}$, and $\alpha$ are $13.1 \mathrm{~K}, 29.9 \mathrm{~K}, 77.9 \mathrm{~K}$, and 2.9 , respectively. The obtained $T_{\mathrm{vc}}$ is consistent with the result measured at dense filaments around RCW 36 (Hill et al. 2011). Initial values of pixel-bypixel SED fitting were applied with the best-fit values obtained from fitting for the global SED. Then, an SED fitting procedure was performed to minimize $\chi^{2}$ at each pixel. As a result, the five-free $\left(T_{\mathrm{c}}, A_{\mathrm{vc}}, A_{\mathrm{c}}, A_{\mathrm{w}}\right.$, and $\left.A_{\mathrm{PAH}}\right)$ and the three-fixed $\left(T_{\mathrm{w}}, T_{\mathrm{vc}}\right.$, and $\left.\alpha\right)$ parameters better reproduce the observed fluxes with pixel-averaged $\chi_{\nu}^{2}$ (two degrees of 

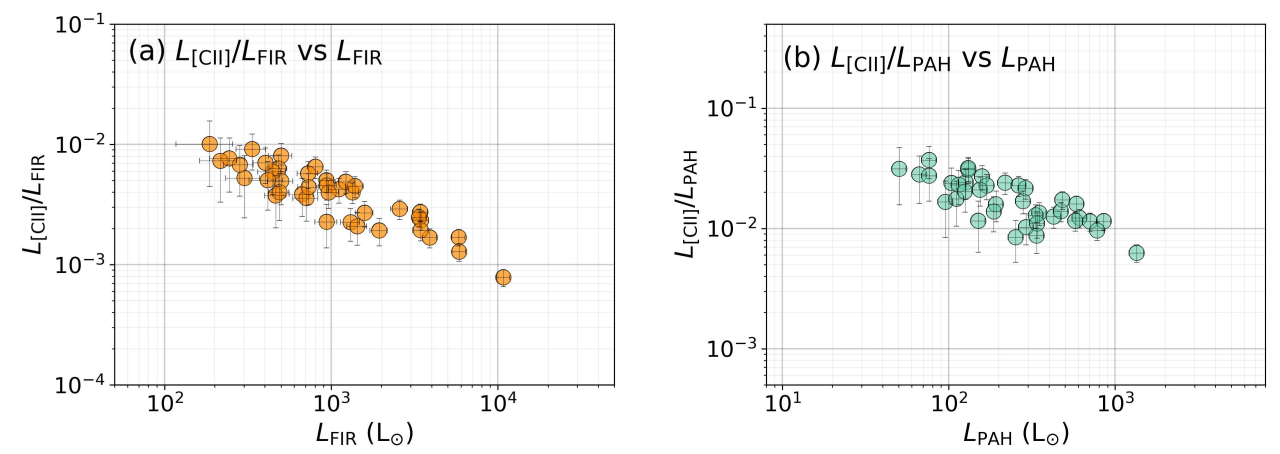

Fig. 8. Correlation plots of (a) $L_{[\mathrm{CII}]} / L_{\mathrm{FIR}}$ vs. $L_{\mathrm{FIR}}$ and (b) $L_{[\mathrm{CII}]} / L_{\mathrm{PAH}}$ vs. $L_{\mathrm{PAH}}$.

freedom, DOF) of 0.4. Resultant pixel-averaged errors on fitted parameters are $3 \%\left(T_{\mathrm{c}}\right), 75 \%\left(A_{\mathrm{vc}}\right), 17 \%\left(A_{\mathrm{c}}\right), 16 \%$ $\left(A_{\mathrm{w}}\right)$, and $12 \%\left(A_{\mathrm{PAH}}\right)$.

Figure 7 shows spatial distributions of cold dust temperature $\left(T_{\mathrm{c}}\right)$, very cold dust, cold dust, warm dust, and PAH luminosities $\left(L_{\mathrm{vc}}, L_{\mathrm{c}}, L_{\mathrm{w}}\right.$, and $\left.L_{\mathrm{PAH}}\right)$ integrated between 3 $1000 \mu \mathrm{m}$ from the best-fit SED model, and the far-IR $\left(L_{\mathrm{FIR}}\right)$ luminsity is calculated from $L_{\mathrm{vc}}+L_{\mathrm{c}}+L_{\mathrm{w}}$. In the case of pixel-by-pixel SED fitting based on the seven-band IR images without the background subtraction, the obtained luminosities are systematically increased by $\sim 1 \%$ along the filament and by $\sim 10 \%$ outside the filament, except for $L_{\mathrm{vc}}$ : $\sim 50 \%$ and $\sim 500 \%$ along and outside the filament, respectively. Because the contribution of $L_{\mathrm{vc}}$ to the total luminosity is significantly smaller than every other components, a factor five uncertainty in $L_{\mathrm{vc}}$ gives a negligible impact on the inferred physical parameters. In the following discussions, the key physical parameters are $L_{[\mathrm{CII}]} / L_{\mathrm{FIR}}$ and $L_{\left[\mathrm{C}_{\text {II }}\right.} / L_{\mathrm{PAH}}$. Since we will discuss an order-of-magnitude variation in those key parameters along and outside the filament regions, the systematic change does not affect our conclusions. Therefore, the luminosities obtained from the background subtraction are applied in the following discussions. The spatial distribution of $T_{\mathrm{c}}$, which is in good agreement with that shown in Hill et al. (2011), shows higher $T_{\mathrm{c}}$ values along the shell-like structures of the bipolar lobe and the ring-like structure caused by the stellar winds from the central OB stars (Minier et al. 2013), while the map shows lower $T_{\mathrm{c}}$ along the filament structure in the north-south direction.

As for luminosity maps, the spatial distribution of $L_{\mathrm{PAH}}$ is very similar to that of $L_{\mathrm{c}}$. The [C II] emission is spatially correlated with both $L_{\mathrm{PAH}}$ and $L_{\mathrm{c}}$, but is relatively poorly correlated with $L_{\mathrm{w}}$ and not for $L_{\mathrm{vc}}$. Overall spatial distributions of $L_{\mathrm{PAH}}$ and $L_{\mathrm{c}}$ show clear shell structures of the bipolar lobe and the ring-like structure, while that of $L_{\mathrm{w}}$ is concentrated around the position of the central OB stars (Ellerbroek et al. 2013). To quantify the spatial correlation, a multiple linear regression analysis was performed using the Python StatsModels package (Seabold \& Perktold 2010). Since $L_{\mathrm{PAH}}$ and $L_{\mathrm{c}}$ are well correlated with each other (correlation coefficient of 0.97 ), both parameters were not included as explanatory variables for the analysis. To make the variance infraction factor (VIF) small, we chose [C II] luminosity, $L_{[\mathrm{C} \text { II] }}$ as the dependent variable, and $L_{\mathrm{PAH}}$ and $L_{\mathrm{w}}$ as explanatory variables. As a result, the adjusted coefficient of determination $R^{2}$ is obtained to be 0.85 with $\mathrm{DOF}=39$ and $\mathrm{VIF}=3.5$, and the correlation to the $L_{\mathrm{PAH}}$ variable is significant $(p<0.001)$, while that to the $L_{\mathrm{w}}$ variable is not $(p=0.47)$ based on the $t$-test with the $95 \%$ confidence level. Given that the PAH emission dominantly comes from PDRs, a good spatial correlation to $L_{\mathrm{PAH}}$ suggests that the [C II] emission dominantly comes from PDRs. The good spatial correlation seen with $L_{\mathrm{c}}$ is also consistent, since the cold dust temperature of $\sim 30 \mathrm{~K}$ is reasonable for a PDR. However, a poor spatial correlation to $L_{\mathrm{w}}$ indicates that the warm dust component mostly trace $\mathrm{H}$ II regions surrounding the OB stars. For the very cold dust component, it is spatially in good agreement with the dense filament structure. The $L_{\mathrm{vc}}$ luminous region around the [CII] emission peak corresponds to the positions of the ${ }^{12} \mathrm{CO}$ peaks and massive clumps identified by Minier et al. (2013). Thus, the very cold dust component well traces dense molecular regions.

\subsection{Variations of $L_{\left[\mathrm{C}_{\mathrm{II}}\right]} / L_{\mathrm{FIR}}$ and $L_{\left[\mathrm{C}_{\mathrm{II}}\right]} / L_{\mathrm{PAH}}$}

In Fig. 8, $L_{\left[\mathrm{C}_{\mathrm{II}}\right]} / L_{\mathrm{FIR}}$ varies about one order of magnitude, while $L_{\left[\mathrm{C}_{\mathrm{II}}\right]} / L_{\mathrm{PAH}}$ shows a smaller variation. Such large variation in $L_{[\mathrm{C} \text { II }} / L_{\mathrm{FIR}}$ is also observed in the Milky Way and nearby galaxies (e.g. Wright et al. 1991; Stacev et al. 1991; Malhotra et al. 2001; Croxall et al. 2012; Kramer et al. 2013; Goicoechea et al. 2015; Smith et al. 2017). According to Goicoechea et al. (2015), the large variation observed toward the Orion molecular cloud 1 (OMC1) can be explained by those in the total dust-cloud column relative to the [C II]-emitting column along each line of sight (geometry effect). To verify whether such geometry effects can also cause a large range of $L_{[\mathrm{CI}]} / L_{\mathrm{FIR}}$ in RCW 36 , the dust opacity close to the [C II $158 \mu \mathrm{m}$ line is calculated as $\tau_{\mathrm{d}, 160}=10^{-26} A_{\mathrm{c}} \nu_{160}^{\beta_{c}} \Omega_{\text {pix }}^{-1}$ for an optically-thin condition, where $\Omega_{\text {pix }}$ is the solid angle subtended by each pixel $\left(90^{\prime \prime}\right)$. Figure 9 shows $L_{[\mathrm{CII}} / L_{\mathrm{FIR}}$ as a function of $\tau_{\mathrm{d}, 160}$, color-coded according to $T_{\mathrm{c}}$. The dashed line corresponds to the relation calculated from a simple face-on slab model assuming a uniform dust temperature: $L_{\left[\mathrm{C}_{\mathrm{II}}\right]} / L_{\mathrm{FIR}}=C\left(1-e^{-\tau_{\mathrm{d}, 160}}\right)^{-1} \simeq C \tau_{\mathrm{d}, 160}^{-1}$ (see Fig. 16a in Goicoechea et al. 2015), where $C$ is a constant determined so that the model curve intercepts the median $L_{[\mathrm{CII}]} / L_{\mathrm{FIR}}$ and $\tau_{\mathrm{d}, 160}$ values. Clearly, the measured data are distributed along the model line because of small variation in the cold dust temperature. Therefore, large variations in $L_{\left[\mathrm{C}_{\mathrm{II}}\right]} / L_{\mathrm{FIR}}$ toward RCW 36 are explained by the geometry effect like the case in OMC1. 


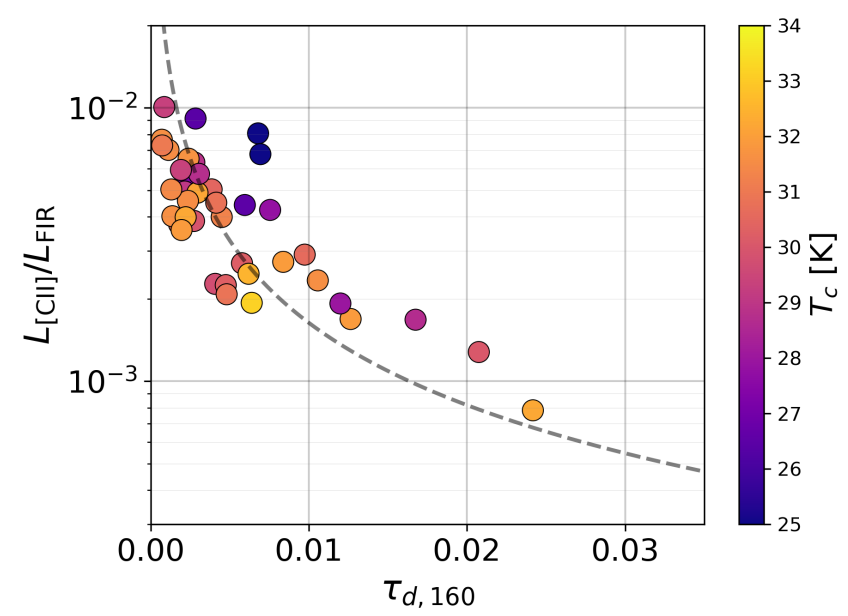

Fig. 9. Correlation plot of $L_{[\mathrm{C} \text { II }} / L_{\mathrm{FIR}}$ vs. $\tau_{\mathrm{d}, 160}$. The dashed line shows the relation with the model of a simple face-on slab of dust with the [C II] foreground emission Goicoechea et al. 2015).

From the spatial correlation analysis among [C II], PAH, warm, cold and very cold dust emissions, [C II] and PAH emissions are spatially in good agreement with each other and thus are considered to mainly arise from the farUV illuminated face of clouds. Then, significant variation in $L_{[\mathrm{CI}]} / L_{\mathrm{PAH}}$ is not expected from the geometry effect. Thus, another factor seems to affect the observed $L_{\left[\mathrm{C}_{\text {II }}\right]} / L_{\mathrm{PAH}}$ variation. In such far-UV dominated regions (PDRs), PAHs are expected to play a significant role of photo-electric heating of gas (Bakes \& Tielens 1994). However, its heating efficiency is decreased as the far-UV radiation field increases due to the presence of larger amount of ionized PAHs (Okada et al. 2013). To verify if photoelectric heating efficiency can explain the observed variation of $L_{\left[\mathrm{C}_{\mathrm{II}}\right]} / L_{\mathrm{PAH}}$, we calculated the intensity of the incident far-UV radiation field $G_{0}$ in Habing units by using the following equation (Hollenbach \& Tielens 1999): $G_{0} \sim I_{\mathrm{FIR}} / 2.6 \times 10^{-4} \mathrm{erg} \mathrm{sec}-1 \mathrm{~cm}^{-2} \mathrm{sr}^{-1}$, where $I_{\mathrm{FIR}}$ is the far-IR intensity obtained from $L_{\text {FIR }}$ at each pixel. $G_{0}$ values thus obtained show the range of $\sim 10^{2}-10^{4}$; the peak $G_{0}$ in RCW36 is $\sim 6$ times lower than that in RCW 38 (Kaneda et al. 2013). As expected, $L_{[\mathrm{C} \text { II }]} / L_{\mathrm{PAH}}$ decreases as $G_{0}$ increases as shown in Fig. 10. Based on this measured variation of $G_{0}$ across RCW 36, we explored the range of values of charging parameter which is relevant for photo-electric heating efficiency. We estimated the charging parameter $\gamma=G_{0} \sqrt{T_{\mathrm{g}}} / n_{\mathrm{e}}$, where $T_{\mathrm{g}}$ and $n_{\mathrm{e}}$ are the gas temperature and the electron density in PDRs. The electron density is calculated from the following equation with assumptions that (a) the electrons in PDRs are all provided by ionized carbons and the carbon atoms are fully ionized $\left(n_{\mathrm{e}}=1.6 \times 10^{-4} n_{\mathrm{H}}\right.$, Sofia et al. 2004) and (b) pressure between H II regions and PDRs is balanced $\left(2 n_{\mathrm{e}, \mathrm{H}}{ }_{\mathrm{II}} T_{\mathrm{e}, \mathrm{H}}\right.$ II $\left.\simeq n_{\mathrm{H}} T_{\mathrm{g}}\right)$;

$n_{\mathrm{e}}=3.2 \times 10^{-4} n_{\mathrm{e}, \mathrm{H} \text { II }}\left(\frac{T_{\mathrm{e}, \mathrm{H} \text { II }}}{T_{\mathrm{g}}}\right)$,

where $n_{\mathrm{H}}, n_{\mathrm{e}, \mathrm{H} I \mathrm{I}}$, and $T_{\mathrm{e}, \mathrm{H}}$ II are hydrogen gas density in PDRs, electron density, and electron temperature in $\mathrm{H}$ II regions, respectively. Moreover, $n_{\mathrm{e}, \mathrm{H} \text { II }}\left(G_{0}\right)$ is derived by solving $G_{0}$ at a distance equal to the Strömgren sphere radius

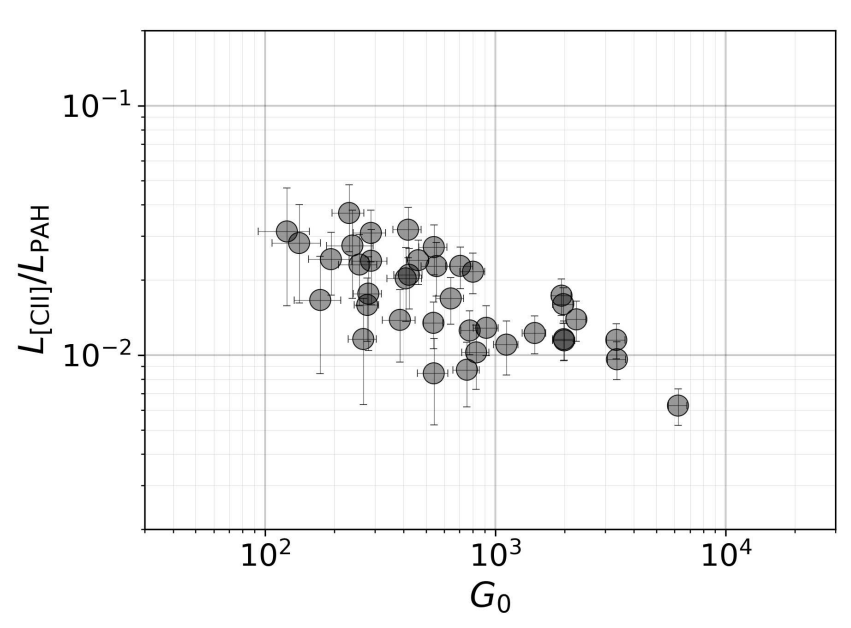

Fig. 10. Correlation plot of $L_{[\mathrm{C} \mathrm{II}]} / L_{\mathrm{PAH}}$ vs. $G_{0}$

and by assuming the fraction of luminosity above $6 \mathrm{eV}$ equal to unity (Tielens 2005) as

$$
\begin{array}{r}
n_{\mathrm{e}, \mathrm{H} \text { II }}\left(G_{0}\right) \simeq 0.78 G_{0}^{\frac{3}{4}}\left(\frac{5 \times 10^{49} \text { photons sec }^{-1}}{N_{\text {Lyc }}}\right)^{-\frac{1}{2}} \\
\times\left(\frac{L_{\star}}{7.6 \times 10^{5} \mathrm{~L}_{\odot}}\right)^{-\frac{3}{4}},
\end{array}
$$

where $N_{\text {Lyc }}$ and $L_{\star}$ are the total number of ionizing photons by a star and the stellar luminosity, respectively. When we apply $N_{\text {Lyc }}=10^{48}$ photons $\mathrm{sec}^{-1}$ for the spectral type of O9 as the dominant exciting star (Binder \& Povich 2018), $L_{\star}=8 \times 10^{4} L_{\odot}$ (Thompson 1984), $T_{\mathrm{e}, \mathrm{H} \text { II }}=$ $7600 \mathrm{~K}$ (Shaver \& Goss 1970), and measured $G_{0}$ values, $\gamma$ ranges from $\sim 10^{3}$ to $10^{5} \sqrt{\mathrm{K}} \mathrm{cm}^{-3}$ for typical $T_{\mathrm{g}}$ of $10^{2}-10^{3} \mathrm{~K}$ in PDRs (Kaufman et al. 1999). The obtained $\gamma$ range meets the transition where the charge state of PAHs changes from neutral to fully ionized states Okada et al. 2013). Therefore, the $L_{\left[\mathrm{C}_{\mathrm{II}}\right]} / L_{\mathrm{PAH}}$ variation can be explained by the variation of the photo-electric heating efficiency on PAHs.

\subsection{Enhanced brightness ratio of $[C I I] / 160 \mu \mathrm{m}$}

In Fig. 4, [C II] $/ 160 \mu \mathrm{m}$ values for RCW 36 are systematically higher than those for $\mathrm{RCW} 38$, while $[\mathrm{C} \mathrm{II}] / 9 \mu \mathrm{m}$ values for RCW 36 are in good agreement with those for RCW 38. Those results indicate that the PAH emission relative to the large-grain emission in RCW 36 is higher than that in RCW 38: difference in SEDs between RCW 36 and RCW 38 .

From the previous two sections, results of pixel-by-pixel correlation analyses for RCW 36 suggest that the $160 \mu \mathrm{m}$ emission traces the column density of dust grains in overall clouds along the line of sight, while [C II] and PAH emissions mainly arise from the far-UV illuminated surface of clouds. RCW 36 is formed in a filamentary molecular cloud with $\mathrm{H}_{2}$ column densities $\left(N_{\mathrm{H}_{2}}\right)$ of $\sim 10^{22}-10^{23} \mathrm{~cm}^{-2}$ Hill et al. 2011). In the outside (east-west side) of the filament, $N_{\mathrm{H}_{2}}$ is rapidly dropped to $\sim 10^{21} \mathrm{~cm}^{-2}$ : low-column density areas of cold gas and dust grains. In this situation, the [C II] map shows that PDRs are formed not only in the 

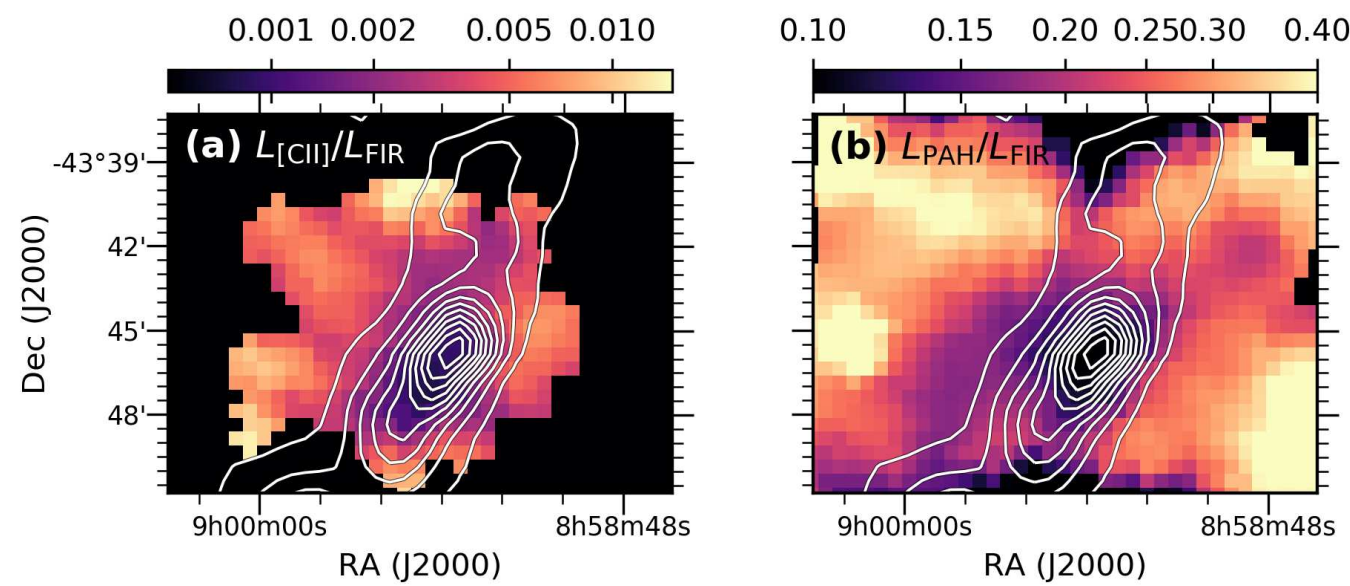

Fig. 11. Luminosity ratio maps of (a) $L_{[\mathrm{CI}]} / L_{\mathrm{FIR}}$ and (b) $L_{\mathrm{PAH}} / L_{\mathrm{FIR}}$ obtained from Fig. 7 The $L_{\mathrm{vc}}$ contours superimposed on the images are linearly spaced 10 levels from 0.95 to $9.5 \mathrm{~L} \odot$

filament but also in low-column density areas because of the bipolar outflow. Therefore, as shown in Fig[11, higher values of $L_{[\mathrm{C} \text { II }]} / L_{\mathrm{FIR}}$ and $L_{\mathrm{PAH}} / L_{\mathrm{FIR}}$ are observed in lowcolumn density areas $\left(\tau_{\mathrm{d}, 160} \lesssim 5 \times 10^{-2}\right)$ rather than in the filament.

Based on the picture of the geometry effect, the overall difference in $[\mathrm{C}$ II $] / 160 \mu \mathrm{m}$ between RCW 36 and RCW 38 can be explained by the difference in the total dust-cloud column relative to the [C II] emitting column. Far-UV photons from massive stars in a filamentary molecular cloud like RCW 36 are likely to leak into low-dust column density regions perpendicular to the filament, while those in a clumpy molecular cloud as in the case of RCW 38 are less likely because stars are surrounded by high-dust column density regions (Wolk et al. 2006; Kaneda et al. 2013; Fukui et al. 2016). In such low-dust column density regions, the [CII] emitting layer is considered to be dominated. Therefore, the enhanced brightness ratio of $[\mathrm{C}$ II $] / 160 \mu \mathrm{m}$ suggests that RCW36 is dominated by far-UV illuminated cloud surfaces (diffuse PDRs) compared with RCW38.

\section{Conclusions}

Large-area [C II] $158 \mu \mathrm{m}$ mapping of RCW 36 was conducted to investigate properties of [C II] emission influenced by a radiative feedback from massive stars formed in the dense filamentary cloud. The [C II] emission overall extends toward the east-west direction from the peak, which is almost perpendicular to the cold dense filament direction. There are two prominent arms extending from the $[\mathrm{CII}]$ peak to the north-east and south-west directions, which are spatially in good agreement with shell-like structures of the bipolar lobe clearly seen in the cold dust $\left(T_{\mathrm{d}} \sim 30 \mathrm{~K}\right)$ and PAH emissions. From a pixel-by-pixel correlation analysis, the $[\mathrm{C}$ II] emission spatially correlates with PAH and cold dust emissions. Therefore, the observed [C II] emission dominantly comes from PDRs. Moreover, we found that the brightness ratio of $[\mathrm{C} \mathrm{II}] / 160 \mu \mathrm{m}$ for RCW 36 is systematically higher than that for RCW 38 , while that of $[\mathrm{C} \mathrm{II}] / 9 \mu \mathrm{m}$ for RCW 36 is consistent with RCW 38. The $L_{\left[\mathrm{C}_{\mathrm{II}}\right]} / L_{\mathrm{FIR}}$ ratio shows large variation $\left(10^{-2}-10^{-3}\right)$ compared with the $L_{\left[\mathrm{C}_{\mathrm{II}}\right]} / L_{\mathrm{PAH}}$ ratio. Given the fact that the tight correlation between $L_{[\mathrm{CI}]} / L_{\mathrm{FIR}}$ and $\tau_{\mathrm{d}, 160}$, the large variation in $L_{\left[\mathrm{C}_{\text {II }}\right]} / L_{\mathrm{FIR}}$ can be explained by that in the total dust-cloud column relative to the $[\mathrm{CII}]$ and PAHs emitting column along each line of sight (geometry effect). Based on the picture of the geometry effect, the enhanced brightness ratio of $[\mathrm{C} \mathrm{II}] / 160 \mu \mathrm{m}$ is attributed to the difference in gas structures where massive stars are formed; unlike RCW 38 where massive stars are formed inside a clumpy molecular cloud, RCW 36 is formed in a filamentary molecular cloud and is then dominated by far-UV illuminated cloud surfaces. Thus, the difference in large-scale gas structures causes the enhanced brightness ratio of [C II] $/ 160 \mu \mathrm{m}$.

Acknowledgements. We greatly appreciate all the members of the Infrared Astronomy Group of TIFR and the staff members of the TIFR Balloon Facility in Hyderabad, India, for their support during balloon flights. We acknowledge support of the Department of Atomic Energy, Government of India, under project no. 12-R\&DTFR-5.02-0200 The authors gratefully acknowledge the contribution of the anonymous referee's comments in improving our manuscript and also thank Dr. Sano for providing contours of CO data. Part of this work is based on observations with AKARI, a JAXA project with the participation of ESA. PACS has been developed by a consortium of institutes led by MPE (Germany) and including UVIE (Austria); KU Leuven, CSL, IMEC (Belgium); CEA, LAM (France); MPIA (Germany); INAF-IFSI/OAA/OAP/OAT, LENS, SISSA (Italy); IAC (Spain). This development has been supported by the funding agencies BMVIT (Austria), ESA-PRODEX (Belgium), CEA/CNES (France), DLR (Germany), ASI/INAF (Italy), and CICYT/MCYT (Spain). SPIRE has been developed by a consortium of institutes led by Cardiff University (UK) and including Univ. Lethbridge (Canada); NAOC (China); CEA, LAM (France); IFSI, Univ. Padua (Italy); IAC (Spain); Stockholm Observatory (Sweden); Imperial College London, RAL, UCL-MSSL, UKATC, Univ. Sussex (UK); and Caltech, JPL, NHSC, Univ. Colorado (USA). This development has been supported by national funding agencies: CSA (Canada); NAOC (China); CEA, CNES, CNRS (France); ASI (Italy); MCINN (Spain); SNSB (Sweden); STFC, UKSA (UK); and NASA (USA). This research is supported by JSPS KAKENHI Grant Numbers JP25247020, JP18H01252.

\section{References}

Anderson, L. D., Zavagno, A., Deharveng, L., et al. 2012, A\&A, 542, A10

André, P., Men'shchikov, A., Bontemps, S., et al. 2010, A\&A, 518, L102

Aniano, G., Draine, B. T., Gordon, K. D., \& Sandstrom, K. 2011, PASP, 123, 1218

Bakes, E. L. O. \& Tielens, A. G. G. M. 1994, ApJ, 427, 822

Baug, T., Ojha, D. K., Dewangan, L. K., et al. 2015, MNRAS, 454, 4335

Binder, B. A. \& Povich, M. S. 2018, ApJ, 864, 136

Bohlin, R. C., Savage, B. D., \& Drake, J. F. 1978, ApJ, 224, 132 
Casey, C. M. 2012, MNRAS, 425, 3094

Croxall, K. V., Smith, J. D., Wolfire, M. G., et al. 2012, ApJ, 747, 81

Dewangan, L. K., Baug, T., Ojha, D. K., et al. 2017a, ApJ, 845, 34

Dewangan, L. K., Ojha, D. K., \& Zinchenko, I. 2017b, ApJ, 851, 140

Draine, B. T. \& Li, A. 2007, ApJ, 657, 810

Ellerbroek, L. E., Bik, A., Kaper, L., et al. 2013, A\&A, 558, A102

Fukui, Y., Torii, K., Ohama, A., et al. 2016, ApJ, 820, 26

Gaia Collaboration, Brown, A. G. A., Vallenari, A., et al. 2018, A\&A, 616, A1

Ghosh, S. K., Iyengar, K. V. K., Rengarajan, T. N., et al. 1988, ApJ, 330,928

Glass, I. S. 1999, Handbook of Infrared Astronomy

Goicoechea, J. R., Teyssier, D., Etxaluze, M., et al. 2015, ApJ, 812, 75

Hill, T., Motte, F., Didelon, P., et al. 2011, A\&A, 533, A94

Hollenbach, D. J. \& Tielens, A. G. G. M. 1999, Reviews of Modern Physics, 71,173

Ishihara, D., Onaka, T., Kataza, H., et al. 2010, A\&A, 514, A1

Jaffe, D. T., Zhou, S., Howe, J. E., et al. 1994, ApJ, 436, 203

Kaneda, H., Nakagawa, T., Ghosh, S. K., et al. 2013, A\&A, 556, A92

Kaufman, M. J., Wolfire, M. G., Hollenbach, D. J., \& Luhman, M. L. 1999, ApJ, 527, 795

Kramer, C., Abreu-Vicente, J., García-Burillo, S., et al. 2013, A\&A, 553, A114

Li, A. \& Draine, B. T. 2001, ApJ, 554, 778

Malhotra, S., Kaufman, M. J., Hollenbach, D., et al. 2001, ApJ, 561, 766

Minier, V., Tremblin, P., Hill, T., et al. 2013, A\&A, 550, A50

Molinari, S., Swinyard, B., Bally, J., et al. 2010, A\&A, 518, L100

Mookerjea, B., Ghosh, S. K., Kaneda, H., et al. 2003, A\&A, 404, 569

Myers, P. C. 2009, ApJ, 700, 1609

Nakagawa, T., Yui, Y. Y., Doi, Y., et al. 1998, ApJS, 115, 259

Okada, Y., Pilleri, P., Berné, O., et al. 2013, A\&A, 553, A2

Onaka, T., Matsuhara, H., Wada, T., et al. 2007, PASJ, 59, 401

Sano, H., Enokiya, R., Hayashi, K., et al. 2018, PASJ, 70, S43

Seabold, S. \& Perktold, J. 2010, in 9th Python in Science Conference

Shaver, P. A. \& Goss, W. M. 1970, Australian Journal of Physics Astrophysical Supplement, 14, 133

Smith, J. D. T., Croxall, K., Draine, B., et al. 2017, ApJ, 834, 5

Sofia, U. J., Wolff, M. J., Rachford, B., et al. 2004, in American Astronomical Society Meeting Abstracts, Vol. 205, 59.16

Stacey, G. J., Geis, N., Genzel, R., et al. 1991, ApJ, 373, 423

Suzuki, T., Kaneda, H., Onaka, T., Nakagawa, T., \& Shibai, H. 2010, A\&A, 521, A48+

Thompson, R. I. 1984, ApJ, 283, 165

Tielens, A. G. G. M. 2005, The Physics and Chemistry of the Interstellar Medium

Wolk, S. J., Spitzbart, B. D., Bourke, T. L., \& Alves, J. 2006, AJ, 132,1100

Wright, E. L., Mather, J. C., Bennett, C. L., et al. 1991, ApJ, 381, 200

Xue, M., Jiang, B. W., Gao, J., et al. 2016, ApJS, 224, 23

Zinnecker, H. \& Yorke, H. W. 2007, ARA\&A, 45, 481 